\title{
Orbitofrontal Cortex Volume and Effortful Control as Prospective Risk Factors for Substance Use Disorder in Adolescence
}

\author{
Ali Cheetham ${ }^{a, b}$ Nicholas B. Allen ${ }^{d, e, g}$ Sarah Whittle $^{f}$ Julian Simmons ${ }^{e, f}$ \\ Murat Yücel ${ }^{c, f}$ Dan I. Lubman ${ }^{a, b}$ \\ ${ }^{a}$ Turning Point, Eastern Health, Fitzroy, Vic., ${ }^{b}$ Eastern Health Clinical School, and ${ }^{\mathrm{c} B r a i n}$ and Mental Health \\ Laboratory, Monash Institute of Cognitive and Clinical Neurosciences, and School of Psychological Science, \\ Monash University, ${ }^{\mathrm{d}}$ Orygen, The National Centre of Excellence in Youth Mental Health, ${ }^{\mathrm{e}}$ Melbourne School \\ of Psychological Sciences, and ${ }^{f}$ Melbourne Neuropsychiatry Centre, Department of Psychiatry, University of \\ Melbourne, Melbourne, Vic., Australia; 9 Department of Psychology, University of Oregon, Eugene, Oreg., USA
}

\section{Key Words}

Substance use disorder · Adolescence .

Structural MRI - Orbitofrontal cortex

\begin{abstract}
Background: Orbitofrontal cortex (OFC) dysfunction has been proposed to increase the risk for developing a substance use disorder (SUD) during adolescence. In this study, we suggest that a reduction in OFC volumes might underlie temperament-based risk factors for SUD, and examined whether smaller OFC volumes during early adolescence could predict later development of SUD. Methods and Materials: Adolescents ( $\mathrm{n}=107 ; 58$ male, 49 female) underwent structural MRI and completed a self-report measure of temperamental effortful control at age 12 . At 3 subsequent assessments (aged 15, 16, and 18) SUD was assessed via a semi-structured clinical interview. By the third assessment, 24 participants $(22.4 \%)$ had received a lifetime diagnosis of SUD. Results: Smaller volumes of the left OFC, right OFC, and left medial subregions predicted lifetime history of SUD by age 18. Volumes of the left OFC and left lateral subregions were positively correlated with effortful control, and left OFC volumes mediated the relationship between effortful control and SUD. Conclusions: Smaller volumes of the OFC
\end{abstract}

(c) 2016 S. Karger AG, Basel

E-Mail karger@karger.com

www.karger.com/ear and low effortful control during adolescence appear to be associated phenotypes that increase the risk of subsequent SUD. Further studies examining the temporal sequence of these risk factors are needed to fully understand this relationship.

(c) 2016 S. Karger AG, Base

\section{Introduction}

It has been proposed that orbitofrontal cortex (OFC) dysfunction represents a neural phenotypic marker of risk for substance misuse in adolescence [1]. Evidence supporting this hypothesis has come from a small number of studies examining the neurobiological correlates of early substance use in adolescent populations [2-5], as well as from studies examining adults with a family history of alcoholism [6,7]. While these findings provide clues about the role of the OFC as a candidate marker of later substance misuse, the actual mechanisms that influence these associations are not well understood. Furthermore, it is yet to be established whether OFC abnormalities can prospectively predict more harmful patterns of use (as opposed to the initiation of use) during this period.
Prof. Dan I. Lubman, MB ChB, PhD

Turning Point

54-62 Gertrude Street

Fitzroy, VIC 3065 (Australia)

E-Mail dan.lubman@monash.edu 
The OFC subserves a number of functions that may explain its association with vulnerability to substance misuse. There is an extensive literature that supports the involvement of this region in encoding and monitoring the reward value of stimuli, and in exercising inhibitory control over reward-related behaviour $[8,9]$. The medial and lateral regions have been proposed to serve different functions in relation to reward and decision-making processes, with the medial region involved in learning and monitoring the reward value of stimuli, and the lateral OFC assisting in the evaluation of negative reinforcement and subsequent adaptation of behaviors. The OFC has also been proposed to form an important component of the neural circuitry underlying temperament, in particular the regulatory dimension of effortful control [10]. Individual differences in effortful control are thought to be established early in life (i.e., 2-3 years of age), and are believed to contribute to the development of aggression, externalizing behavior problems, and substance misuse in adolescence [11]. Accordingly, low effortful control has been found to predict the onset and progression of substance use $[12,13]$, and has been strongly associated with adolescent substance use disorder (SUD) [14]. It is thus likely that the OFC forms an important component of the neural circuitry underlying self-regulatory abilities, which may help to explain the link between effortful control and risk for substance misuse.

Studies examining the OFC as a potential marker of vulnerability to substance use and misuse have used varied methodologies and populations that have differed markedly across studies in relation to age and risk status. Nevertheless, there has been some consistency in findings, most notably among structural imaging studies that have found smaller OFC volumes (particularly in the right hemisphere $[2,5,7]$ ) appearing to be associated with earlier onset of use in adolescents, and vulnerability to alcoholism in adults. In 2 of these studies [5, 7], right OFC volume reductions were found to co-occur or correlate with high impulsivity (a lower-order trait related to effortful control), suggesting that a reduction in OFC volume could underlie temperament traits associated with risk. However, this hypothesis is yet to be tested using an adequate design (i.e., prospective) or appropriate analysis (i.e., formal statistical tests of mediation). Prospective assessment is of particular importance, given that heavy substance use can contribute to OFC dysfunction [15], and may disrupt frontal cortical development during adolescence [16, 17]. While cross-sectional analyses can provide useful information about risk when substance use is still minimal (e.g., [4]), a prospective, longitudinal approach is needed in order to examine the contribution of OFC abnormalities to more harmful levels of use.

Recently, we demonstrated that smaller volumes of the OFC at age 12 predicted the initiation of cannabis use by age 16 [2]. This study represents the analysis of data from a subsequent wave of assessment at age 18 from the same sample, with the aims of (i) determining whether volume reductions predict the more harmful patterns of use that typically emerge later in adolescence; and (ii) investigating whether the link between OFC volume reductions and substance use risk may be associated with temperamental effortful control. As the relationship between OFC dysfunction and risk is unlikely to be substance specific [1], and in recognition of the high rates of comorbidity between different SUDs [18], we broadened our previous focus from cannabis to include all substances. Specifically, we hypothesised that smaller OFC volumes at age 12 would predict lifetime history of SUD by age 18. In addition, given the aforementioned research linking the OFC to temperamental risk factors for substance misuse, we hypothesised that smaller OFC volumes would mediate the relationship between low effortful control (also measured at age 12) and later SUD.

\section{Methods and Materials}

\section{Participants}

Participants were recruited from schools across metropolitan Melbourne, Australia, as part of a larger study on adolescent development (the ORYGEN Adolescent Development Study), which has been described in detail previously [19]. The selection procedure for the larger study involved administering the Early Adolescent Temperament Questionnaire - Revised (EATQ-R [20]) to participants during class time, for the purpose of selecting a smaller sample $(n=415)$ that represented a broad spectrum of risk for later onset of psychopathology (for further detail on recruitment and inclusion/exclusion criteria, see [19]). Specifically, we recruited an equal number of male and female participants who had scores that were $0-1,1-2,2-2.5$, and greater than 2.5 SDs above and below the mean for the EATQ-R temperament dimensions of negative affectivity and effortful control. This resulted in a smaller sample that over-represented adolescents with scores in the tails, but retained the range in temperament seen in the larger screening sample. Of those selected, 245 consented to participate in one or more phases of more intensive research.

At the baseline assessment, 155 participants (mean age \pm SD $12.7 \pm 0.5$ years) underwent structural MRI, a semi-structured clinical interview (the Schedule for Affective Disorders and Schizophrenia for School-Aged Children; K-SADS [21]), and were again administered the EATQ-R. No participants were found to have evidence of alcohol or other SUD at this time point. At 3 subsequent follow-up assessments, the full sample of 245 participants were invited to complete the K-SADS ( $\mathrm{n}$ at the first follow-up = 211 , mean age $15.0 \pm 0.43$ years; $n$ at the second follow-up $=172$,
38

Eur Addict Res 2017;23:37-44 DOI: $10.1159 / 000452159$
Cheetham/Allen/Whittle/Simmons/ Yücel/Lubman 
mean age $16.6 \pm 0.60 ; \mathrm{n}$ at the third follow-up $=161$, mean age $18.8 \pm 0.5$ years). Data from these assessments were used to establish lifetime diagnoses of alcohol and other SUD by the third follow-up assessment. In total, 107 participants who completed the K-SADS at the third follow-up assessment had also provided MRI data at baseline. There were no significant differences in OFC volumes between these participants and those who provided MRI data but did not participate in the third follow-up assessment $(\mathrm{n}=$ 46 ; right $\mathrm{OFC} \mathrm{t}=-0.395$, d.f. $=151, \mathrm{p}=0.694$; left $\mathrm{OFC} \mathrm{t}=-0.098$, d.f. $=151, p=0.922)$. Similarly, there were no differences between these participants and the full sample of 245 in terms of EC $(t=-0.596$, d.f. $=243, p=0.552)$ or gender $\left(\chi^{2}=0.107, p=0.744\right)$.

\section{Measures}

MRI Image Acquisition and Morphometric Analysis

Scans were acquired using a 3-T scanner at the Brain Research Institute, Melbourne, Australia (see [22] for specifications). Regions of interest were manually traced using ANALYZE software (Mayo Clinic, Rochester, N.Y., USA; http://www.mayo.edu/bir/) and segmented into grey matter, white matter, and cerebrospinal fluid using an automated segmentation tool (FSL) [23]. An estimate of whole brain volume (WBV) was obtained by summing gray and white matter pixel counts (i.e., cerebral grey and white matter, the cerebellum, and the brainstem). Using the method outlined by Riffkin et al. [24], a line through the AC-PC was used to define the superior boundary of the OFC. The posterior boundary of the OFC was marked by a coronal plane passing through the most posterior aspect of the olfactory sulcus in each hemisphere. The inferior boundary was defined by the most inferior aspect of the cortex, the lateral boundary by the most lateral edge of the cortex, and the medial boundary of each hemisphere by the longitudinal fissure. Medial and lateral OFC regions were separated using the medial orbital sulcus. All images were manually edited to eliminate subcortical tissue and artifacts related to the eye sockets and nasal bones. Intra- and inter-rater reliabilities were calculated for each raw volume on a separate set of 10 images, and were acceptable for all regions ( $>0.98$ for overall left and right volumes, and lateral subregions; $>0.75$ for medial subregions). All regions were corrected for WBV, using the covariance approach outlined by Free et al. [25].

\section{Substance Use}

Diagnoses of substance abuse and dependence were assessed using the K-SADS at the 3 follow-up assessments described above. The K-SADS uses DSM-IV criteria to classify symptoms of abuse and dependence as 'not present', 'sub-threshold', or 'threshold'. The age of first regular use was also established using the K-SADS. At the third K-SADS assessment, participants also completed a questionnaire (the Youth Risk Behaviour Survey; YRBS [26]) that assessed lifetime use of alcohol and cannabis (specific questions included 'during your life, on how many days have you had at least one drink of alcohol?' and 'during your life, on how many days have you used (illicit substance)?'), as well as past-year experience of alcohol-related problems [27] and past-year binge drinking (i.e., consumption of 5 or more standard drinks in one session).

\section{Socioeconomic Status}

A measure of socioeconomic status (SES) was derived using data on parental occupation at baseline. Occupations were coded according to the ANU_4 scale [28], a socioeconomic index derived specifically for the Australian context that classifies occupation

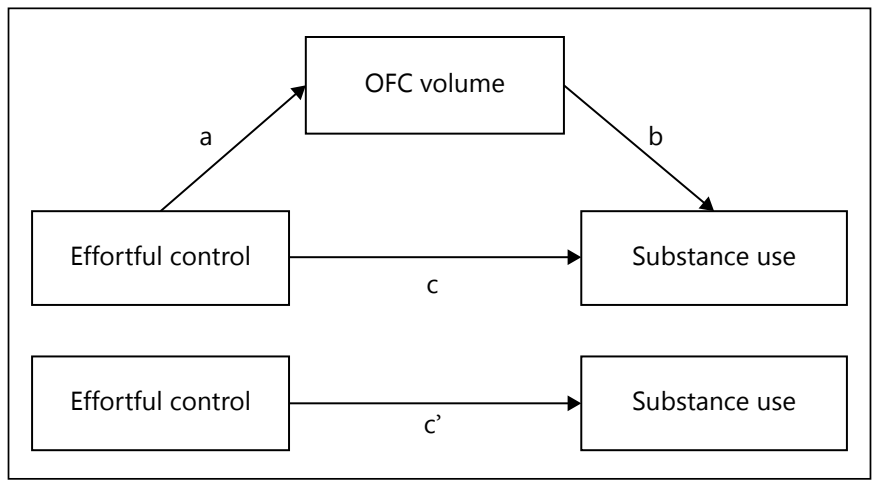

Fig. 1. Proposed mediation model of the influence of effortful control at age 12 on substance use at age 18 , via OFC volume at age 12 . Path a is the total effect of effortful control on OFC volume, path $b$ is the total effect of OFC volume on substance use, path $c$ is the total effect of effortful control on substance use (i.e., not controlling for OFC volume), and path c' is the direct effect of effortful control on substance use (i.e., controlling for OFC volume).

based on social and economic characteristics. For parents with missing occupational data, a measure of education was substituted (total years in school, scaled to reflect ANU_4 codes).

\section{Intelligence}

The fourth version of the Wechsler Intelligence Scale for Children was used to assess intelligence (IQ) at baseline. Estimated full-scale IQ (FSIQ) scores were derived using scores on the verbal comprehension, matrix reasoning, and symbol search subtests, in accordance with the methods described in [29]. FSIQ scores from a later assessment (conducted approximately 2.5 years after the baseline assessment) were used for 6 participants who had missing data at the baseline assessment.

\section{Statistical Analysis}

Multinomial logistic regression was used to examine whether OFC volume at baseline was associated with lifetime history of SUD at follow-up. All analyses controlled for gender. Mediation analyses were performed using macros developed by Preacher and Hayes [30], which generate bootstrap CIs for total and direct effects. Mediation was tested by assessing the significance of the cross product of the coefficients for the independent variable (IV; effortful control) to mediator (OFC volume) relation (the path a), and the mediator to dependent variable (DV; SUD) relation controlling for the IV (the path $\mathrm{b}$ ). The $\mathrm{a}-\mathrm{b}$ cross product (the indirect effect) tests the statistical significance of the difference between the total effect, or path c, and the direct effect, or path c', which is the impact of the IV on the DV adjusting for the effect of the mediator (fig. 1) [31]. This approach offers an advantage over Baron and Kenny's 1986 method [32] as it allows for the examination of mediation in the absence of a direct effect of the IV on the DV. Examining indirect pathways even when a significant direct pathway is not detected has been recommended, particularly in longitudinal research, as the total effect is the sum of many different paths of influence (both direct and indirect), and these may cancel each other out if they operate in opposite directions (i.e., if one mediator acts to suppress the other; see [33-35]). Where necessary, me- 
Table 1. Characteristics of substance use groups

\begin{tabular}{|c|c|c|c|c|}
\hline & $\begin{array}{l}\text { Group 1: never experienced } \\
\text { symptoms }(n=44)\end{array}$ & $\begin{array}{l}\text { Group 2: experienced symptoms } \\
\text { but not disorder }(n=43)\end{array}$ & $\begin{array}{l}\text { Group 3: Met criteria for } \\
\text { disorder }(n=20)\end{array}$ & $\mathrm{p}$ value $\mathrm{b}^{\mathrm{b}}$ \\
\hline \multicolumn{5}{|l|}{ KSADS symptoms, mean (SD) } \\
\hline Alcohol & 0 & $1.47(0.73)$ & $3.90(2.55)$ & $<0.001$ \\
\hline Cannabis & 0 & $0.14(0.41)$ & $2.40(3.08)$ & $<0.001$ \\
\hline Other illicit substances ${ }^{\mathrm{a}}$ & 0 & 0 & $3.00(2.82)$ & $<0.001$ \\
\hline Alcohol-related problems & $0.40(0.94)$ & $1.76(1.45)$ & $3.55(2.28)$ & $<0.001$ \\
\hline Binge drinking episodes & $4.91(9.91)$ & $20.00(21.63)$ & $46.63(43.96)$ & $<0.001$ \\
\hline Lifetime days cannabis use & $0.27(0.90)$ & $3.73(8.03)$ & $49.06(81.14)$ & $<0.001$ \\
\hline \multicolumn{5}{|l|}{ Temperament } \\
\hline Effortful control $^{\mathrm{d}}$ & $3.43(0.62)$ & $3.50(0.66)$ & $3.38(0.58)$ & 0.781 \\
\hline \multicolumn{5}{|l|}{ Demographics } \\
\hline SES $^{\mathrm{d}}$ & $58.55(20.90)$ & $60.87(18.33)$ & $53.55(19.32)$ & 0.391 \\
\hline
\end{tabular}

diation analysis also controlled for variables that different significantly across the 3 substance use groups.

Separate analyses were conducted to examine whether WBV was associated with lifetime history of SUD at follow-up, and whether WBV mediated the relationship between effortful control and SUD. Again, all analyses controlled for gender, and where necessary, other variables associated with substance use.

\section{Results}

By the third follow-up assessment, 20 participants had met criteria for past or current SUD. This included 12 participants who had met criteria for alcohol use disorder only ( 9 for abuse, 3 for dependence), 1 participant who had met criteria for cannabis use disorder only (dependence), and 7 participants who had met criteria for both alcohol and cannabis use disorders ( 3 for alcohol and cannabis abuse, 2 for alcohol abuse and cannabis dependence, and 2 for alcohol and cannabis dependence). All participants in the SUD group were assessed as having an onset after the baseline assessment (i.e., after age 12).

The remaining 87 participants were heterogeneous with regard to their level of substance use and associated symptoms. Therefore, they were split into 2 groups in order to capture any effects associated with this variation.
The first group $(n=43)$ included participants who had experienced at least one past or present threshold symptom of SUD (e.g., consumes more than planned; continued use despite recurrent negative physical consequences), but had never met criteria for diagnosis of abuse or dependence. The second group $(n=44)$ included participants who had never experienced any past or present threshold symptoms. No participants reported regular use before 14 years of age, for any substance.

Table 1 summarises the substance use and demographic differences between the 3 groups. There were significant differences between substance use groups with regard to KSADS symptoms (total, alcohol-specific, and cannabis-specific) as well as YRBS lifetime use of alcohol and cannabis, past-year experience of alcohol-related problems, and past-year binge drinking. There were no significant differences between groups in regard to effortful control, gender, age, or SES, although there was a trend for IQ to be higher among participants who had never experienced SUD symptoms. Subsequent analyses therefore controlled for IQ in addition to gender. Table 2 presents mean OFC volumes for each region, following correction for WBV.

OFC volumes demonstrated a linear relationship with substance use group, with the no-symptoms group
40

Eur Addict Res 2017;23:37-44 DOI: $10.1159 / 000452159$
Cheetham/Allen/Whittle/Simmons/ Yücel/Lubman 
Table 2. Means and SDs of regional brain volumes at age 12 measured in $\mathrm{mm}^{3}$ (OFC volumes are presented following correction for WBV)

\begin{tabular}{ccrr}
\hline & $\begin{array}{l}\text { Group 1: never experienced } \\
\text { symptoms }(\mathrm{n}=44)\end{array}$ & $\begin{array}{l}\text { Group 2: experienced symptoms } \\
\text { but not disorder }(\mathrm{n}=43)\end{array}$ & $\begin{array}{l}\text { Group 3: met criteria for } \\
\text { disorder }(\mathrm{n}=20)\end{array}$ \\
\hline Right OFC & $20,898.59(3,142.41)$ & $20,224.38(3,145.75)$ & $19,228.22(3,687.46)$ \\
Right lateral & $13,537.01(2,031.84)$ & $13,291.27(2,308.74)$ & $12,665.92(2,668.86)$ \\
Right medial & $7,498.86(1,557.53)$ & $6,970.16(1,504.36)$ & $6,540.00(1,489.55)$ \\
Left OFC & $20,492.69(3,244.33)$ & $19,562.92(3,815.18)$ & $18,438.93(3,722.41)$ \\
Left lateral & $12,909.71(2,220.02)$ & $12,448.27(2,900.98)$ & $11,542.18(2,766.39)$ \\
Left medial & $7,829.73(1,881.88)$ & $7,190.24(1,379.18)$ & $6,841.58(1,617.19)$ \\
\hline
\end{tabular}

demonstrating the largest volumes and the SUD group demonstrating the smallest volumes (fig. 2). Multinomial logistic regression, controlling for gender and $\mathrm{IQ}^{1}$, revealed that the odds of being in the SUD group (group 3 ), relative to the no-symptoms group (group 1), were increased by smaller volumes of the right OFC (OR $1.020, \mathrm{p}=0.026,95 \%$ CI 1.002-1.038), left OFC (OR $1.021, \mathrm{p}=0.012,95 \% \mathrm{CI} 1.005-1.038)$, as well as right medial (OR 1.046, $\mathrm{p}=0.022,95 \%$ CI $1.007-1.087$ ), and left medial subregions (OR 1.047, $\mathrm{p}=0.016,95 \% \mathrm{CI}$ 1.009-1.087). There were trend-level associations between substance use group and left lateral subregions (OR 1.021, p $=0.058,95 \%$ CI 0.999-1.043) and left medial subregions (OR 1.021, p $=0.097,95 \%$ CI 0.9961.046). Volume differences between the some-symptoms group (group 2) and the SUD (group 3) and nosymptoms groups (group 1) were not significant for any brain regions.

WBV measurements were similar between the nosymptoms group (mean 1,297,979.54; SD 121,543.94), the some-symptoms group (mean 1,287,843.91; SD 106,047.70) and the SUD group (mean 1,319,254.67; SD 104,000.73), with multinomial logistic regression analysis (controlling for gender and IQ) demonstrating no significant differences in volume.

Left OFC volumes demonstrated a significant positive correlation with effortful control $(\mathrm{r}=0.208, \mathrm{p}=0.032)$. This relationship was significant for left lateral regions $(\mathrm{r}=0.234, \mathrm{p}=0.015)$ but not left medial regions $(\mathrm{r}=0.087$, $\mathrm{p}=0.375)$. Overall, right $\mathrm{OFC}$ volumes were not correlated with effortful control $(\mathrm{r}=0.156, \mathrm{p}=0.110)$. There was no relationship between effortful control and WBV volume, controlling for gender $(r=0.124, p=0.202)$.

\footnotetext{
${ }^{1}$ For all analyses, gender was not a significant predictor. However, lower IQ consistently increased the odds of being in the SUD group, relative to the nosymptoms group (ORs between $1.057-1.062$, all $\mathrm{p}$ values $<0.029$ ).
}

OFC Volume and Control as Prospective

Risk Factors for SUD in Adolescence

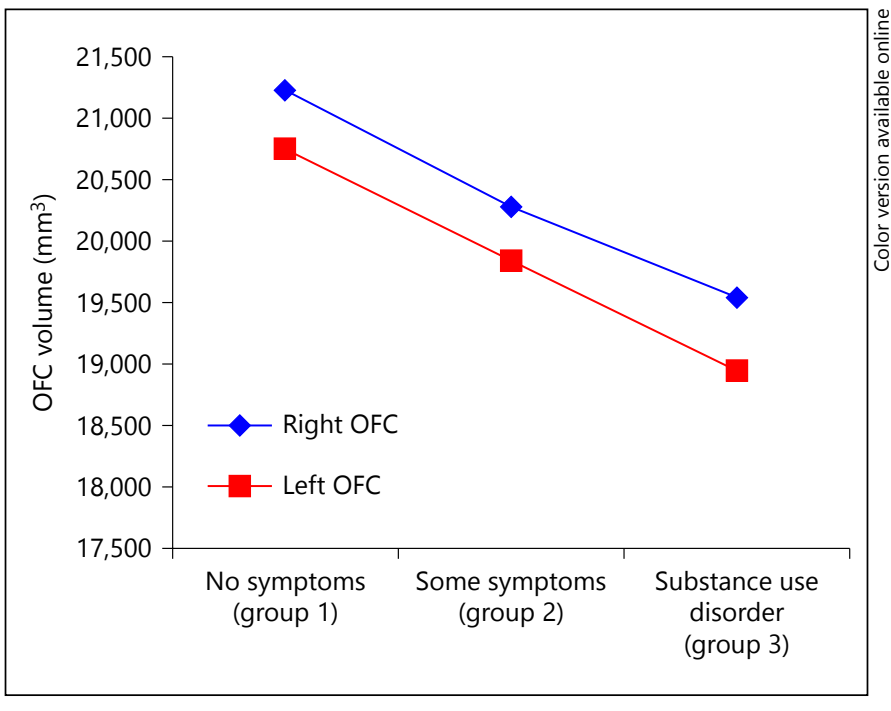

Fig. 2. Corrected overall right and left OFC volumes at age 12 as a function of substance use group at age 18 .

Mediation analyses were performed only for OFC regions that were significantly related to effortful control (indicating a significant path a); thus, right OFC volumes were not examined as potential mediators. Analyses examining the left OFC and left lateral OFC as mediators, controlling for the effects of gender and IQ, are reported in table 3. The direct effect of effortful control on substance use group was not significant. However, there was a significant effect of effortful control on left OFC volumes, with lower levels of effortful control predicting smaller volumes, and a significant effect of left OFC volume on the substance use group, whereby smaller volumes predicted more problematic substance use. Bias-corrected 95\% CIs demonstrated that smaller left OFC volumes significantly partially mediated the relationship between effortful control and substance use. 
Table 3. Mediation analyses examining the relationship between effortful control at age 12 and SUD group at age 18, via left overall OFC and left lateral OFC volumes at age 12

\begin{tabular}{lrrlll}
\hline Mediator & Path a & Path b & Path c' (direct effect) & Path c (total effect) & Indirect effect \\
\hline Left OFC & & & & & \\
$\mathrm{b}$ & 13.625 & -0.006 & 0.165 & 0.867 & mean $=-0.079$ \\
$\mathrm{SE}$ & 5.798 & 0.002 & 0.119 & 0.120 & $\mathrm{SE}=0.005$ \\
$\mathrm{t}$ & 2.350 & -2.972 & 1.388 & -1.404 & upper CI $=-0.200$ \\
$\mathrm{p}$ & 0.021 & 0.004 & 0.168 & 0.472 & lower CI $=-0.011$ \\
\hline Left lateral OFC & & & & & \\
$\mathrm{b}$ & 11.144 & -0.006 & 0.154 & 0.087 & mean $=-0.068$ \\
SE & 4.127 & 0.003 & 0.122 & 0.120 & $\mathrm{SE}=0.042$ \\
$\mathrm{t}$ & 2.700 & -2.15 & 1.262 & 0.472 & upper CI $=-0.179$ \\
$\mathrm{p}$ & 0.008 & 0.034 & 0.210 & & lower CI $=-0.006$ \\
\hline
\end{tabular}

\section{Discussion}

While OFC dysfunction has been well established in addicted populations, more recent research has demonstrated that individual differences in the structure and function of this region are also associated with the early stages of substance use in healthy adolescents. The current study adds to these findings by demonstrating that smaller volumes of the OFC, measured at age 12, prospectively predict SUD by age 18 . This finding was observed for overall right and left OFC volumes, and for medial (but not lateral) subregions in both hemispheres. Analyses also revealed a positive association between overall left $\mathrm{OFC}$ volume and the temperament dimension of effortful control. Furthermore, left OFC volume partially mediated the relationship between effortful control and SUD, even though the direct association between these variables was not significant.

These findings add to a growing literature indicating that alterations in the structure of the OFC represent a marker of vulnerability to substance misuse. To date, these studies have primarily been cross sectional, limiting the inferences that can be made regarding causality (e.g., [5]), and/or have utilized samples with minimal substance use histories, which are unable to provide information on the relationship between OFC structure and more problematic patterns of use (e.g., [2, 4]). The current study provides the first evidence for a prospective association between OFC volume and SUD, extending our previous findings [2], and highlighting the importance of this region in understanding vulnerability to addiction as well as early use. In addition, the results of the mediation analysis provide preliminary support for the neurodevelopmental model of addiction vulnerability described by Zucker et al. [36], in which a pre-existing dysfunction in the neural system that underlies effortful control abilities contributes to the development of SUD during adolescence and early adulthood. Thus, the findings are consistent with previous research indicating that OFC alterations may be associated with temperament-based risk factors for addiction $[4,5,7,37]$.

While the relationship between left OFC volume and effortful control provides support for our hypothesis, the non-significant associations between effortful control and SUD is surprising, given that inhibitory control deficits have been found to robustly predict substance use during adolescence. However, it should be noted that direct effects are not always observable over a long followup period [38], and the presence of a significant indirect effect suggests that effortful control may still have an influence, albeit in a mediated way. Previous research provides some insight into the other variables that could be influencing the relationship between effortful control and substance use. For example, 2 studies found that the association between effortful control and alcohol use was dependent on other risk factors, including exposure to a 'substance using lifestyle' (encompassing personal and peer involvement with alcohol [12]); and reward responsiveness [39]. In both studies, no direct effect of effortful control on alcohol use was evident, but moderating effects were reported; according to these reports, low effortful control was a risk factor among adolescents with minimal exposure to alcohol use and adolescents with high reward responsiveness. It is also possible that the relationship between low effortful control and substance use differs according to the outcome examined, as 2 studies have found it predicts problems with cannabis and other illicit substances but notalcohol use or alcohol-related problems $[12,40]$.
Cheetham/Allen/Whittle/Simmons/ Yücel/Lubman 
Effortful control was also not associated with right OFC volumes, suggesting that alterations in this region may influence vulnerability via some other pathway. As there is evidence that smaller right OFC volume is associated with impulsivity in substance-using and high-risk populations $[5,7,41]$, it is possible that pre-existing differences in this region increase the risk of SUD via an associated increase in this trait. Impulsivity is a complex construct that is believed to arise from a failure in inhibitory control mechanisms, and comprises multiple dimensions that have been strongly associated with various aspects of substance use [42]. Indeed, it has been proposed that the OFC may represent a neural substrate for substance misuse in adolescence due to its role in impulsive behaviour [1]. However, as the measure of effortful control used in the current study is more closely associated with disinhibition than impulsivity [43], it may not have captured the aspects of impulsivity that could account for the association between right OFC volume and risk for SUD.

When subregions of the OFC were examined separately, only the medial regions remained significant predictors of SUD. The medial and lateral regions of the OFC play an important role in the processing of rewards and punishments, respectively, and dysfunction in the medial OFC has been hypothesized to underlie the hypersensitivity to reward and impairment in reward-based decision-making that characterises addiction [44]. Potentially, the association between medial OFC volumes and SUD could reflect pre-existing differences in reward sensitivity that increase the risk of heavier or more frequent substance use during adolescence. It is interesting to note, however, that the pattern of findings in the current study differ from those we reported previously, in which both medial and lateral regions predicted early cannabis use [2]. One explanation for these findings could be that the lateral regions underlie functions associated with substance use initiation, while the medial regions underlie functions associated with heavier or more problematic use. Although speculative, this suggestion is in line with a study by Bolla et al. [45], in which hypoactivation in the lateral OFC during a reward-based decision-making task differentiated cannabis users from controls, while hypoactivation in the medial OFC differentiated heavy from moderate users.

Thelongitudinaldesign of this study isa notable strength, as it allowed for the examination of OFC volume prior to the onset of regular substance use. However, a number of issues and limitations should be acknowledged. Of note, there was variation within the SUD group with regard to problem severity (i.e., abuse versus dependence), as well as overlap between the experience of alcohol and cannabis use problems, with 7 participants (35\%) receiving multiple diagnoses, and we did not have the statistical power to examine these groups separately. While comorbidity between alcohol and other SUDs is to be expected (and it is not uncommon for studies to combine cases of abuse and dependence [18]), being unable to explore potential differences between subgroups of participants limits the conclusions that can be drawn from the findings. For example, the differential relationships that have been observed between effortful control and alcohol and cannabis use in previous studies $[12,40]$ suggest that risk mechanisms for problems associated with different substances may differ. A further limitation is that we did not collect measures of other traits that have been associated with both OFC volume and SUD, such as impulsivity or reward responsiveness. In light of the limited associations that were observed between OFC volume and effortful control, the inclusion of a wider range of measures would allow a more detailed investigation of why individual differences in the OFC are associated with an increased risk of SUD. Finally, there was a trend towards higher IQ among participants with no SUD symptoms, although it should be noted that IQ was controlled for in all subsequent analyses that included OFC volume and effortful control as predictors.

In conclusion, the results of this study provide further evidence that alterations in the OFC are associated with risk for substance misuse in adolescence. It appears that a reduction in OFC volume could underlie the temperament traits that have previously been associated with substance abuse. More broadly, it may be that the brain mechanisms involved in SUD also predispose adolescents to problematic use. As such, our findings have important implications for studies examining the role of the OFC in addiction, and highlight the need for prospective, longitudinal research to examine this issue.

\section{Acknowledgements and Financial Disclosures}

This work was supported by grants from the Colonial Foundation, the National Health and Medical Research Council Australia (NHMRC Program Grant 350241), and the Australian Research Council (Discovery Grants DP0878136, DP120102313). S.W. was supported by a NHMRC Career Development Fellowship (ID 1007716). M.Y. was supported by a NHMRC Senior Researcher Fellowship (ID 1021973).

\section{Disclosure Statement}

All authors have no conflicts of interest to disclose. 


\section{References}

1 Whelan R, Weierstall K, Garavan H: The orbitofrontal cortex, drug use and impulsivity: can teenage rebellion be predicted through neural correlates? Future Neurol 2012;7:507509.

2 Cheetham A, et al: Orbitofrontal volumes in early adolescence predict initiation of cannabis use: a 4-year longitudinal and prospective study. Biol Psychiatry 2012;71:684-692.

3 Lotfipour S, et al: Orbitofrontal cortex and drug use during adolescence: role of prenatal exposure to maternal smoking and BDNF genotype. Arch Gen Psychiatry 2009;66: 1244-1252.

4 Whelan R, et al: Adolescent impulsivity phenotypes characterized by distinct brain networks. Nat Neurosci 2012;15:920-925.

5 Churchwell JC, Lopez-Larson M, YurgelunTodd DA: Altered frontal cortical volume and decision making in adolescent cannabis users. Front Psychol 2010;1:225.

6 Andrews MM, et al: Individuals family history positive for alcoholism show functional magnetic resonance imaging differences in reward sensitivity that are related to impulsivity factors. Biol Psychiatry 2011;69:675-683.

7 Hill SY, et al: Disruption of orbitofrontal cortex laterality in offspring from multiplex alcohol dependence families. Biol Psychiatry 2009;65:129-136.

8 Kringelbach ML, Rolls ET: The functional neuroanatomy of the human orbitofrontal cortex: evidence from neuroimaging and neuropsychology. Prog Neurobiol 2004;72:341372.

9 Sescousse G, et al: Processing of primary and secondary rewards: a quantitative meta-analysis and review of human functional neuroimaging studies. Neurosci Biobehav Rev 2013;37:681-696.

10 Whittle $\mathrm{S}$, et al: The neurobiological basis of temperament: towards a better understanding of psychopathology. Neurosci Biobehav Rev 2006;30:511-525.

11 Rothbart MK, et al: Developing mechanisms of temperamental effortful control. J Pers 2003;71:1113-1143.

12 Piehler TF, Véronneau MH, Dishion TJ: Substance use progression from adolescence to early adulthood: effortful control in the context of friendship influence and early-onset use. J Abnorm Child Psychol 2012;40:10451058.

13 Creemers HE, et al: Predicting life-time and regular cannabis use during adolescence; the roles of temperament and peer substance use: the TRAILS study. Addiction 2010;105:699708 .

14 Willem L, et al: Temperamental characteristics of adolescents with substance abuse and/ or dependence: a case-control study. Pers Individ Dif 2011;50:1094-1098.

15 Goldstein RZ, Volkow ND: Dysfunction of the prefrontal cortex in addiction: neuroim- aging findings and clinical implications. Nat Rev Neurosci 2011;12:652-669.

16 Crews F, He J, Hodge C: Adolescent cortical development: a critical period of vulnerability for addiction. Pharmacol Biochem Behav 2007;86:189-199.

17 Bava S, Tapert SF: Adolescent brain development and the risk for alcohol and other drug problems. Neuropsychol Rev 2010;20:398413.

18 Kessler RC, et al: Prevalence, severity, and comorbidity of 12-month DSM-IV disorders in the National Comorbidity Survey Replication. Arch Gen Psychiatry 2005;62:617-627.

19 Yap MB, Allen NB, Sheeber L: Using an emotion regulation framework to understand the role of temperament and family processes in risk for adolescent depressive disorders. Clin Child Fam Psychol Rev 2007;10:180-196.

20 Ellis LK, Rothbart MK: Revision of the Early Adolescent Temperament Questionnaire. Minneapolis, Poster Presented at the 2001 Biennial Meeting of the Society for Research in Child Development, 2001.

21 Kaufman J, et al: Schedule for affective disorders and schizophrenia for school-age children-present and lifetime version (K-SADSPL): initial reliability and validity data. J Am Acad Child Adolesc Psychiatry 1997;36:980988.

22 Whittle S, et al: Neuroanatomical correlates of temperament in early adolescents. J Am Acad Child Adolesc Psychiatry 2008;47:682-693.

23 Zhang Y, Brady JM, Smith SM: An HMRF-EM Algorithm for Partial Volume Segmentation of Brain MRI: FMRIB Technical Report TR01YZ1. Technical Report, Oxford Centre for Functional Magnetic Resonance Imaging of the Brain, 2001.

24 Riffkin J, et al: A manual and automated MRI study of anterior cingulate and orbito-frontal cortices, and caudate nucleus in obsessivecompulsive disorder: comparison with healthy controls and patients with schizophrenia. Psychiatry Res 2005;138:99-113.

25 Free SL, et al: Methods for normalization of hippocampal volumes measured with MR. AJNR Am J Neuroradiol 1995;16:637-643.

26 CDC: Youth Risk Behavior Surveillance System. Atlanta, Centers for Disease Control and Prevention, 1999.

27 Little K, et al: Longitudinal predictors of alcohol-related harms during the transition to adulthood. Aust Psychol 2013;48:270-280.

28 Jones FL, McMillan J: Scoring occupational categories for social research: a review of current practice, with Australian examples. Work Employ Soc 2001;15:539-563.

29 Sattler JM, Dumont R: Assessment of Children: WISC-IV and WPPSI-III Supplement. San Diego, Jerome M. Sattler, Publisher, 2004.

30 Preacher KJ, Hayes AF: SPSS and SAS procedures for estimating indirect effects in simple mediation models. Behav Res Methods Instrum Comput 2004;36:717-731.

31 Whittle S, et al: Pituitary volume mediates the relationship between pubertal timing and depressive symptoms during adolescence. Psychoneuroendocrinology 2012;37:881-891.

32 Baron RM, Kenny DA: The moderator-mediator variable distinction in social psychological research: conceptual, strategic, and statistical considerations. J Pers Soc Psychol 1986; 51:1173-1182.

33 Collins LM, Graham JJ, Flaherty BP: An alternative framework for defining mediation. Multivariate Behav Res 1998;33:295-312.

34 MacKinnon DP, et al: A comparison of methods to test mediation and other intervening variable effects. Psychol Methods 2002;7:83104.

35 Hayes AF: Beyond Baron and Kenny: Statistical Mediation Analysis in the NewM. Communication Monographs, 2009, vol 76, pp 408-420.

36 Zucker RA, Heitzeg MM, Nigg JT: Parsing the undercontrol/disinhibition pathway to substance use disorders: a multilevel developmental problem. Child Dev Perspect 2011;5: 248-255.

37 Hill SY, et al: Temperament at 5 years of age predicts amygdala and orbitofrontal volume in the right hemisphere in adolescence. Psychiatry Res 2010;182:14-21.

38 Shrout PE, Bolger N: Mediation in experimental and nonexperimental studies: new procedures and recommendations. Psychol Methods 2002;7:422-425.

39 Willem L, Bijttebier P, Claes L: Reactive and self-regulatory temperament dimensions in relation to alcohol use in adolescence. Addict Behav 2010;35:1029-1035.

40 Wong MM, Rowland SE: Self-determination and substance use: is effortful control a mediator? Alcohol Clin Exp Res 2013;37:1040-1047.

41 Crunelle CL, et al: Reduced frontal brain volume in non-treatment-seeking cocaine-dependent individuals: exploring the role of impulsivity, depression, and smoking. Front Hum Neurosci 2014;8:7.

42 Dawe S, Gullo MJ, Loxton NJ: Reward drive and rash impulsiveness as dimensions of impulsivity: implications for substance misuse. Addict Behav 2004;29:1389-1405.

43 Rothbart MK, et al: Investigations of temperament at three to seven years: the Children's Behavior Questionnaire. Child Dev 2001;72: 1394-1408.

44 Bechara A, Damasio H: Decision-making and addiction (part I): impaired activation of somatic states in substance dependent individuals when pondering decisions with negative future consequences. Neuropsychologia 2002; 40:1675-1689.

45 Bolla KI, et al: Neural substrates of faulty decision-making in abstinent marijuana users. Neuroimage 2005;26:480-492. 